\title{
Olivier Abel, Paul Ricœur, Jacques Ellul, Jean Carbonnier, Pierre Chaunu - Dialogues
}

Genève, Labor et Fides, 2012, 136 p.

\section{Daniel Vidal}

\section{(2) OpenEdition}

\section{Journals}

Édition électronique

URL : http://journals.openedition.org/assr/24228

DOI : $10.4000 /$ assr. 24228

ISSN : $1777-5825$

Éditeur

Éditions de l'EHESS

Édition imprimée

Date de publication : 30 décembre 2012

ISSN : 0335-5985

\section{Référence électronique}

Daniel Vidal, «Olivier Abel, Paul Ricœur, Jacques Ellul, Jean Carbonnier, Pierre Chaunu - Dialogues », Archives de sciences sociales des religions [En ligne], 160 | octobre-décembre 2012, mis en ligne le 21 février 2013, consulté le 21 septembre 2020. URL : http://journals.openedition.org/assr/24228 ; DOI https://doi.org/10.4000/assr.24228

Ce document a été généré automatiquement le 21 septembre 2020.

(c) Archives de sciences sociales des religions 


\section{Olivier Abel, Paul Ricœur, Jacques Ellul, Jean Carbonnier, Pierre Chaunu - Dialogues}

Genève, Labor et Fides, 2012, 136 p.

Daniel Vidal

\section{RÉFÉRENCE}

Olivier Abel, Paul Ricœur, Jacques Ellul, Jean Carbonnier, Pierre Chaunu - Dialogues, Genève, Labor et Fides, 2012, 136 p. 
1 Quatre figures de "protestants illustres » parmi les penseurs contemporains issus de, ou venus à, la Réforme. Tous, auteurs d'une œuvre considérable, malaisément définie en termes de disciplines, qu'elle transgresse au contraire allègrement. Paul Ricœur (1913-2005), philosophe, herméneute, moraliste; Jacques Ellul (1912-1994), déchiffreur du monde moderne, cet «empire du non-sens » en sa dérive technicienne; Jean Carbonnier (1908-2003), analyste intransigeant du droit et de la loi comme «mal

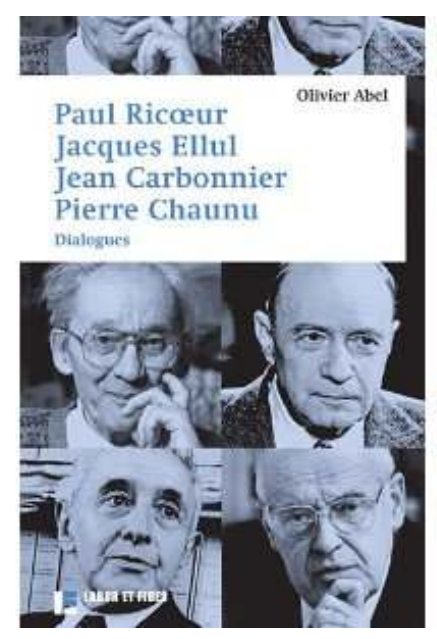
nécessaire »; Pierre Chaunu (1923-2009), historien des Amériques, de l'Europe des Lumières, de la Réforme, et d'une «brève histoire de Dieu ». Dans cette constellation d'œuvres et de personnalités, définir des axes de croisements, des surfaces de recouvrements : telle fut l'intention d'Olivier Abel, qui regroupe aujourd'hui la matière première d'entretiens télévisés enregistrés en 1992-1994. Au risque, entremêlant anecdotes et fragments d'analyses, questions en archipel au fil du discours, de « divertir » le lecteur plus que de l'engager à approfondir les leçons de ces maitres. Demeurent cependant des lignes de force, ou lignes de crête, qui, dans le cadre privilégié par l'auteur, d'une intime adhésion au protestantisme, ressortissent à la Réforme en sa plus exigeante "pensée » - la plus grande fidélité, remarque Abel, passant le plus souvent par la transgression, le « débordement ». Et l'on sait en effet, combien chacun, en ses compétences propres, et ses aires d'analyse, a su mettre en intrigue des arguments et des questions s'ouvrant au plus grand large. Si l'on veut définir ce qui pourrait valoir pente commune de la pensée protestante, ce serait sans doute en cette passion du sens multiple, loin de toute positivité d'un savoir en absolu, et, dans la pluralité du signe, la chance de pouvoir ouvrir en permanence de nouveaux champs d'intelligibilité. "Passion pour le possible », remarque l'auteur, qui vient de cette capacité à saisir dans un même mouvement de la pensée, ce qui, dans l'exégèse biblique, par exemple, mais cela vaut aussi pour un questionnement plus radicalement "profane", relève de la tension eschatologique et de la raison institutionnelle. Du prophétisme ou de la mystique, et de la régulation de la loi. Ou, pour reprendre l'exemple proposé par Olivier Abel, de la centralité de l'État, et de la résistance à l'irrationalité du politique. C'est dire que chaque auteur ici sollicité, fut en son entier habité d'une passion personnelle du savoir, et pleinement présent au monde. Quand Marx demandait que l'on passe de l'interprétation du monde à sa transformation, posant une coupure entre ces deux modalités d'intervention, il s'agit ici au contraire de maintenir l'un et l'autre de ces impératifs en un même horizon d'action.

2 Si P. Ricœur pense le plus directement la question de mal, chacun s'y confronte dans le déploiement même de son analyse. Déplacer le problème du mal, et de la faute qui le précède comme son ombre, vers la souffrance, cette violence faite à l'innocence, cette « fatalité » dont Job est la figure fondamentale dans l'univers de la Bible, c'est proposer une histoire qui serait enfin celle des victimes et des vaincus, après qu'elle le fut des bourreaux et des vainqueurs. C'est, du même coup, reprendre à nouveaux frais le 
thème de la responsabilité, celle qui relève du sujet et de son action personnelle dans le monde, à l'égard d'autrui ou de la règle du jeu social. Jean Carbonnier veut que des limites soient apportées au principe de responsabilité et, selon sa belle expression, que "le droit donne la parole au temps». Et que, restreinte, à terme elle soit éteinte, le sujet ainsi rapatrié dans le monde commun des justifiés. Des vulnérables, dont $\mathrm{H}$. Arendt demandait que le politique assure un minimum de protection, par l'efficacité même de son exercice. Tout sujet est "fragile ", toute loi est «flexible", toute action humaine est fondamentalement structurée par un conflit. Son paradigme le plus déterminant, dans la conception "protestante ", liera l'ordre de la justice rétributive, qui dit la dépendance de chaque sujet avec tout autre, selon le schème anthropologique du don et du contre-don - et la logique de pure gratuité de l'amour, qui ne requiert nulle réciprocité, mais l'entier désinvestissement de soi, dont témoigne tout le mouvement mystique, cette face cachée de la Réforme qu'il faudra bien un jour mettre en aveu, quand le catholicisme la surexpose, pensant la maitriser.

Une autre complicité s'avère entre les quatre œuvres résumées par Olivier Abel: la question de temps. Inscrite à même la "promesse », chez Ricœur, qui implique du destinataire l'attente de son accomplissement, et pose une relation entre temps et altérité ; reprise par Pierre Chaunu et réinterprétée, dans sa profondeur historique, aux fins de "construire un passé qui serve à notre présent»; définie, on l'a vu, par Carbonnier, comme principe d'allégement de la responsabilité et, à terme, de sa résorption, donnant ainsi chance à tout sujet singulier de demeurer sujet social; reformulée par J. Ellul comme question chaque fois concernant l'homme sans qualité, " sans grand $\mathrm{H}$ ", au-delà de toute abstraction, au-delà de toute allégorie : comme ce qui fait présence et présent, dans les communautés de quelques-uns, capables seules, disait Gide, de "sauver le monde ». Au reste, le salut est « une affaire qui nous est donnée » et, revenant à Calvin comme à la source la plus claire, il ne faut pas avoir souci de sa justification, qui serait alors "marque du péché ", de n'être pas «entièrement vidé de soi ». Le temps traverse ainsi, et travaille, chaque œuvre, et la même question ouvre des espaces de significations profondément bouleversés-bouleversants. Pourrait-il en aller autrement, si l'on se rappelle ceci, de Saint-Augustin, évoqué par Pierre Chaunu : «Toi qui vois le temps depuis l'éternité, sais-tu ce que c'est de voir le temps depuis le temps, combien c'est pénible?».

4 De la grâce, il est dit qu'elle est à raison d'existence, et que c'est sous condition désormais de telle radicale gratuité que tout sujet peut jubiler de se savoir enfin libre. De son «destin", ce choix pleinement assumé. Chaunu dit cette grâce libératoire, Carbonnier la dit prise d'autonomie d'un sujet «abandonné au libre jeu de ses facultés, responsable de son interprétation même ». C'est sans doute en le double foyer de la Réforme, que cette "liberté », et cette joie, se déploient. Luther au cœur de tous les troubles de l'esprit et de la volonté, de la souffrance d'exister, de Dieu toujours en son absence, « ouvre la brèche ", et dit la seule solution, à vrai dire la plus simple : l'amour. Calvin ne connaît pas tels désarrois de l'âme, mais, juriste, veut une société " ordonnée ». La grâce est la conjonction immédiate de l'amour pur et du devoir d'être toujours "soi-même comme un autre », selon la formule de P. Ricœur. Ainsi la grâce, propose $\mathrm{O}$. Abel, n'est-elle plus « réponse au péché, mais au néant ».

5 D'un thème à l'autre, chaque auteur peut alors trouver en tout autre bien plus qu'un miroir de ses propres pensées: une lecture et une écriture s'ouvrant à d'autres dimensions de l'histoire et du sujet en son existence singulière, attestant de la rigueur 
d'un même "style " protestant de quête et de disponibilité à l'incertitude du savoir. Entre ces quatre figures de la pensée Réformée contemporaine, quelque chose comme une symphonie. Pastorale? 